\title{
Effects of Glutamine and Omega-3 Fatty Acids on Erythrocyte Deformability and Oxidative Damage in Rat Model of Enterocolitis
}

\author{
Ruksan Cehreli ${ }^{\mathrm{a}, \mathrm{e}}$, Hale Akpinar ${ }^{\mathrm{b}}$, Aysegul Temiz Artmann ${ }^{\mathrm{c}}$, Ozgul Sagol ${ }^{\mathrm{d}}$
}

\begin{abstract}
Background: The aim of the study was to investigate preventive effects of glutamine (Gln), omega-3 fatty acids (FA) on erythrocyte deformability (EDEF) in rat model of indomethacin-induced enterocolitis.

Methods: Nineteen Wistar albino male rats were divided into three groups: control group, colitis induced by indomethacin and were fed with a standard laboratory diet (group 1), and colitis induced by indomethacin and were also fed with Gln, omega-3 FA (group 2). An investigation was performed in a rat model of experimental colitis induced by subcutaneous injections of $2 \mathrm{~mL}$ intdomethacine solution applied at 24 and 48 hours intervals to male Wistar rats for 14 days. Gln and omega-3 FA were added to the daily standard diets of the animals during 14 days of injections. During the study, changes in body weight were evaluated. The intestines were examined, and colitis was macroscopic and histologically scored. The circulating tumor necrosis factor alpha (TNF- $\alpha$ ) and interleukine-1 $\beta$ (IL-1 $\beta$ ), erythrocyte transit time (ETT) and thiobarbituric acid reactive substances (TBARS) levels were determined in addition to calculation of EDEF indices in all groups.
\end{abstract}

Results: No significant differences in body weight changes could be determined between the standard diet and special diet groups at the end of the experiment. After macroscopic and microscopic scoring, in all of the groups that colitis was found induced, the lowest microscopic score was observed in the group 2. But Gln and omega-3 FA supplemented diet did not change the mean macroscopic and histological

Manuscript accepted for publication September 24, 2015

aDepartment of Prevantive Oncology, Institute of Oncology, Dokuz Eylul University Inciralti, Izmir 35340, Turkey

bDivision of Gastroenterology, Dokuz Eylul University School of Medicine, Inciralti, Izmir 35340, Turkey

'Department of Cell Biophysics and Cellular Engineering, Institute for Bioengineering, Aachen University of Applied Sciences, Germany

${ }^{\mathrm{d}}$ Department of Pathology, Dokuz Eylul University School of Medicine, Inciralti, Izmir 35340, Turkey

${ }^{e}$ Corresponding Author: Ruksan Cehreli, Department of Prevantive Oncology, Institute of Oncology, Dokuz Eylul University Inciralti, Izmir 35340, Turkey. Email: ruksan.cehreli@deu.edu.tr

doi: http://dx.doi.org/10.14740/gr683w scores in all rats. The proliferating cell nuclear antigen (PCNA) levels were significantly higher in group 1 and group 2 compared to the control group. Effects of the diet on circulating TNF- $\alpha$ and IL- $1 \beta$ levels were found correlated with inflammation but statistically significant differences were not found in the group 1 and group $2(\mathrm{P}<0.05)$. The ETT and TBARS levels in standard and special diet groups were significantly increased $(\mathrm{P}<0.05)$. However, EDEF indices which are an important parameter of the study were decreased in indomethacininduced enterocolitis groups that fed with standard and special diet.

Conclusions: Increases in ETT and TBARS levels did not return to normal by addition of Gln and omega-3 FA to diet. Our results suggest that determination of effective optimal doses and route of administration for these nutrients may play an important role in reducing EDEF and microvascular changes.

Keywords: Erythrocyte deformability; Oxidative stress; Inflammatory bowel disease; Enterocolitis; Glutamine; Omega-3 fatty acids

\section{Introduction}

Inflammatory bowel disease (IBD) occurs as a result of the combined effects of environmental changes, multiple genetic variations, oxidative stress, alterations in intestinal microflora and abberrations in innate and adaptive immune responses [1]. Local inflammatory mediators such as eicosanoids, proinflammatory cytokines and reactive oxygen species (ROS) play an important role in the pathogenesis of the disease [2-5]. The impairment of the gut barrier function is also the common characteristic of IBD. It may be due to changes of bacterial microflora, excessive immune cell mediated response and immune cell infiltration in the intestinal wall. These events would result in intestinal tissue destruction and the developement of IBD due to increased ROS and cytokine production [6].

Also ROS attacks may also cause membrane protein oxidation and lipid peroxidation. Erythrocyte membranes are vulnerable to lipid peroxidation because of the lipid components of their membranes.

Lipid peroxidation has adverse effects on the deformability of erythrocytes [7].

Deceleration in microvascular flow is another important factor in the etiopathogenesis of IBD which may associate with dis- 
torted erythrocyte deformability (EDEF) [8]. Although EDEF has been an intensively studied in chronic diseases, but only one clinical IBD study has been reported in the literature revealing that increased erythrocyte malonyldialdehyde content causes a reduction in EDEF [9]. In IBD microvascular flow slowdown can be expected to induce decrease in EDEF, but data on EDEF are insufficient.

Anti-inflammatory therapies are beneficial against active IBD. There is also evidence that a number of nutrients may act to suppress inflammation [10].

In recent years, nutrition has emerged as an important part of IBD therapy.

Glutamine (Gln) is a multifunctional amino acid and is also the main fuel for rapidly dividing cells such as enterocytes, colonocytes, immune cells such as lymphocytes ( $\mathrm{T}$ and B cells) and macrophages [11-14]. Gln has also been considered as a candidate for barrier functions and antioxidant therapy in laboratory models [6].

Omega-3 fatty acids (FA) are precursors of potent lipid mediators, termed eicosanoids, and they have anti-inflammatory properties. Omega-3 FA have also been shown to alter the production of inflammatory cytokines [15] and have local and systemic supressive effects on cell mediated immunity via cytokine release (including tumor necrosis factor alpha (TNF- $\alpha$ )) [16].

The aim of this study was to evaluate the influence of a Gln and omega-3 FA supplemented diet on inflammation, EDEF, erythrocyte oxidative damage, and serum TNF- $\alpha$ and interleukine-1 $\beta$ (IL-1 $\beta$ ) levels in rat model of indomethacininduced enterocolitis.

\section{Materials and Methods}

\section{Animals model}

Care and handling of the animals were in compliance with the internationally accepted standard guidelines for use of animals, and approval was obtained from the animal ethics council of Dokuz Eylul University School of Medicine (DEUSM).

DEUSM Experimental Research Laboratory provided 19 Wistar albino male rats weighing $200-300 \mathrm{~g}$ used in this study. The rats were housed in standard single cages in a room under constant temperature $22 \pm 2{ }^{\circ} \mathrm{C}, 55 \pm 5 \%$ humidity, and a $12 \mathrm{~h}$ light/dark cycle at the DEUSM Experimental Animal Laboratory.

Experimental animals were divided into three groups.

The control group included five rats that were not treated with indomethacin and each of the other two groups that were treated with indomethacin included seven rats.

All animals were given standard and specific diets according to the experimental design (lab diet; Oriental Yeast Company, Tokyo, Japan) and water ad libitum.

All rats were fasted for $16 \mathrm{~h}$, and weight gains were registered before the procedure of laparotomy.

\section{Induction of colitis}

All rats were put in a special cage where they could fit comfortably. Enterocolitis (intestinal inflammation) was induced by indomethacin at a dose of $7.5 \mathrm{mg} / \mathrm{kg}$ dissolved in $5 \%$ sodium bicarbonate given in two subcutaneous injections at 24 $\mathrm{h}$ interval $(\mathrm{n}=7)$. The $0.5 \mathrm{~mL}$ physiological saline was subcutaneously administered to the rats in the control group $(n=5)$.

This represents a rat model for Crohn's disease [16]. After indomethacin administration, no rats developed perforation due to the formation of ulcerations in the colon. However, indomethacin-induced gastrointestinal bleeding in rats were not counted because of exclusion.

\section{Experimental design}

Nineteen Wistar albino male rats were divided into three groups . The rats in the control group $(n=5)$ were not treated with indomethacin and were fed on standard laboratory diet (lab diet; Oriental Yeast Company, Tokyo, Japan).

Group $1(n=7)$ : the rats were fed with a standard laboratory diet (lab diet; Oriental Yeast Company, Tokyo, Japan), and group $2(n=7)$ : in addition to standard diet, the rats were also fed with a special diet supplemented Gln $(12.5 \% \mathrm{mg})$, omega-3 FA ( $8.5 \%$ g) (Oriental Yeast Company, Tokyo, Japan).

The rats received standard chow diet or a special diet for 14 days between the day before the first indomethacin injection and the fasting period before the laparatomy procedure. Their body weights were recorded daily.

\section{Histopathologic examination}

After 14 days, $24 \mathrm{~h}$ of fasting blood samples were drawn from the abdominal aorta under ether anesthesia, and then the rats were sacrificed because of hypovolemia. Decapitation was performed after tissue samples were collected for pathological examination. The abdominal cavity was opened by a midline incision. The entire length of small bowel and colon, from the pylorus to the rectum was then removed. The lumen was gently flushed with phosphate-buffered saline $(0.9 \% \mathrm{NaCl})$ for the clearance of fecal material. The lumen was then opened longitudinally, and fresh specimen was examined under a microscope at a magnification of $\times 5$ by a pathologist who was blind to the experiment. The extent of mucosal damage was assessed by using the macroscopic inflammatory scoring (MIS) system of Wallace et al [17]. Following evaluation of macroscopic lesions, tissue sections were obtained containing the areas of gross ulcerative lesions, including the normal mucosa next to the lesions.

The tissues were fixed in formaldehyde, embedded in paraffin, and tissue sections stained with hematoxylin and eosin. Histopathologic evaluations were made under a light microscope by the same experienced pathologist who was again blind to the experiment. The lesions were scored as described by Vilaseca et al [18].

A standard streptavidin biotin immune-peroxidase method was used for proliferating cell nuclear antigen (PCNA) immunostaining (dilution: 1:100). The tissue sections were deparaffinized and rehydrated, and endogenous peroxidase activity was blocked using a $0.3 \%$ solution of hydrogen peroxide in phosphate buffered saline (PBS) at room temperature for $10 \mathrm{~min}$. The sections were then boiled in citrate buffer solution in a mi- 
Table 1. Changes in Body Weight in the Experimental Groups

\begin{tabular}{llll}
\hline Groups & Baseline weight & Final weight & Difference in body weight \\
\hline Control $(\mathrm{n}=5)$ & $235.8 \pm 19.3$ & $248.9 \pm 17.9$ & 13.1 \\
Standard diet $(\mathrm{n}=7)$ & $244.5 \pm 8.7$ & $174.3 \pm 6.8$ & -3.4 \\
$\begin{array}{l}\text { Special diet (supplemented with } \\
\text { Gln + omega-3 FA) }(\mathrm{n}=7)\end{array}$ & $270 \pm 12.1$ & $266.2 \pm 10.3$ & -4.2 \\
\hline
\end{tabular}

The results are shown as the mean \pm SD.

crowave oven, three times for ten min for epitope retrieval. Primary antibodies were applied for $30 \mathrm{~min}$ at room temperature, and the slides were washed in TRIS buffer. Linking antibody and streptavidin peroxidase complex (LSAB kit) were added consecutively for $10 \mathrm{~min}$ at room temperature again followed by washing in TRIS buffer. Appropriate tissue sections were simultaneously stained with the primary antibody as positive controls. The most representative areas of the section, including mucosa next to the ulcers, were selected and marked for analysis. Antibody staining results were scored semiquantitatively.

\section{Serum TNF- $\alpha$ and IL-1 $\beta$ levels}

Serum TNF- $\alpha$ and IL- $1 \beta$ levels were measured using a sensitive commercially available rat specific enzyme-linked immunosorbent assay (ELISA) kit following the manufacturer's instructions (Endogen Inc., Woburn, MA, USA).

\section{Evaluation of erythrocyte thiobarbituric acid reactive substances (TBARS) and ETT}

EDEF is the average duration of the passage of 1,000 erythrocytes through $5 \mu \mathrm{m}$ diameter pores in milliseconds assessed using a cell transit analyzer (CTA). Venous blood samples anticoagulated with heparin were analyzed to assess the EDEF within a 30 min sampling time using a CTA. Breifly, a 20\% suspension of washed erythrocytes was passed through nucle- opore polycarbonate membranes with a $15 \mathrm{~mm}$ diameter and one $5 \mu \mathrm{m}$ diameter pore. An increased cell transit time is reflected by a decreased EDEF [19-22].

Lipid peroxides were estimated using the method of Stock, Dormandy and Jain which measures TBARS [23, 24].

\section{Statistical analyses}

All data are expressed as the mean \pm SD (SEM). Comparisons between groups of non-parametric data were performed using the Mann-Whitney U test. Statistical analysis was performed using SPSS 15.0 software. Statistical significance was designated for $\mathrm{P}$ value $<0.05$.

\section{Results}

The rats in the control group gained an average of $13.1 \mathrm{~g}$ in weight, and the rats in the standard diet and special diet groups lost 3.4 and $4.2 \mathrm{~g}$, respectively. No significant differences in body weight changes could be determined between group 1 and group 2 at the end of the experiment (Table 1).

\section{Histopathologic findings of intestine}

Administration of indomethacin resulted in clear damage in the ileum and notably also in the proximal part of the colon.

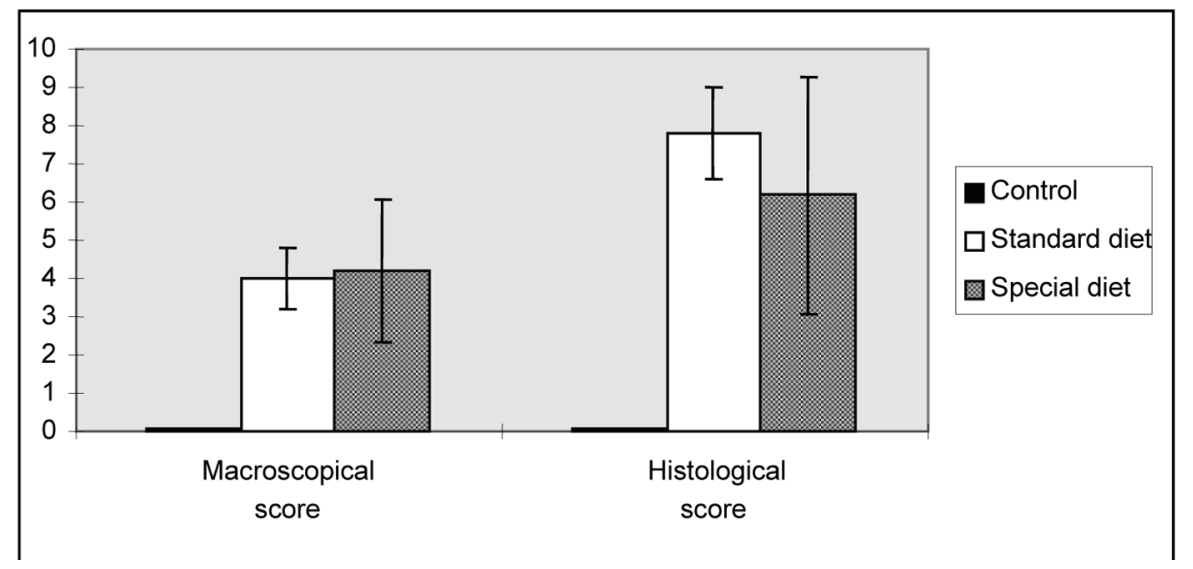

Figure 1. Morphological lesion scores for the intestinal damage induced by indomethacin. The results are shown as the mean \pm SD. 


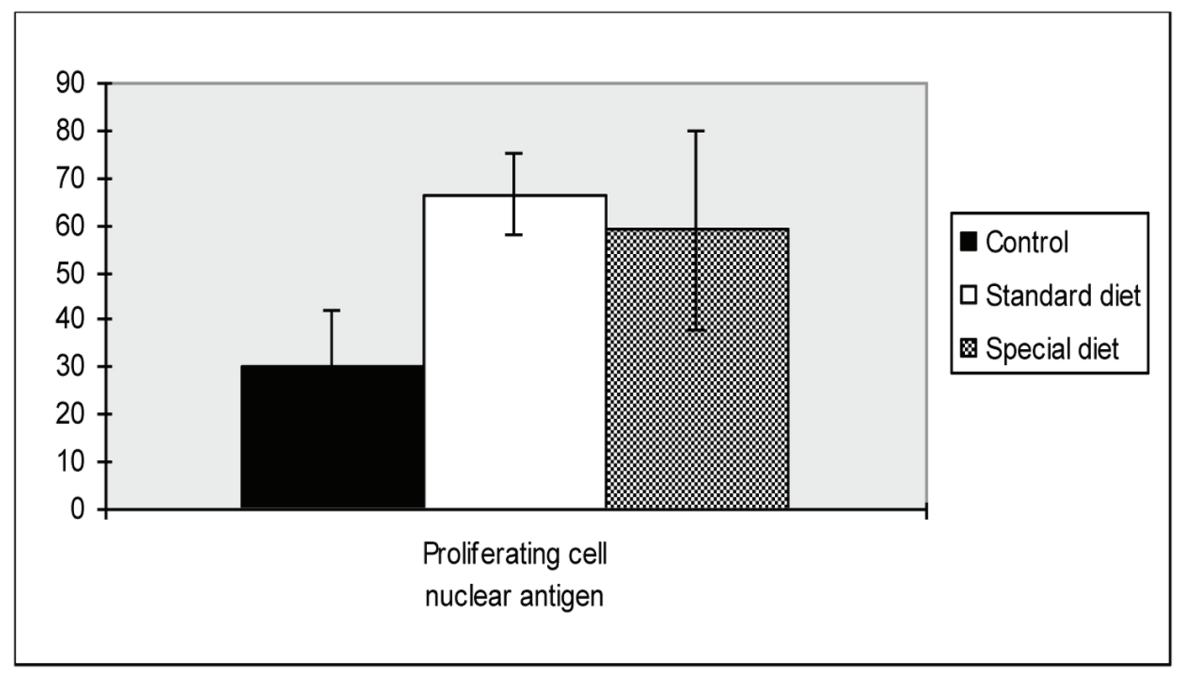

Figure 2. Proliferating cell nuclear antigen scores of intestine. Mean values are shown. ${ }^{*} \mathrm{P}<0.05$ compared to the control group.

By 14 days after indomethacin administration, distinct areas of ulceration and inflammation separated by regions of grossly normal mucosa were detectable.

Macroscopic ulceration was not observed in the control group. Upon macroscopic examination, a $15 \mathrm{~mm}$ ulcer in the intestinal tissue was observed in one rat in the standard diet group, while three and four larger ulcers $(>2 \mathrm{~cm})$ were observed in group 1 and group 2, respectively. After macroscopic and microscopic scoring, in all of the groups that colitis was found induced, the lowest microscopic score was observed in the group 2. Although these scores were significantly higher in rats fed on standard and special diets compared to rats in the control group. The standard and special diets did not alter the

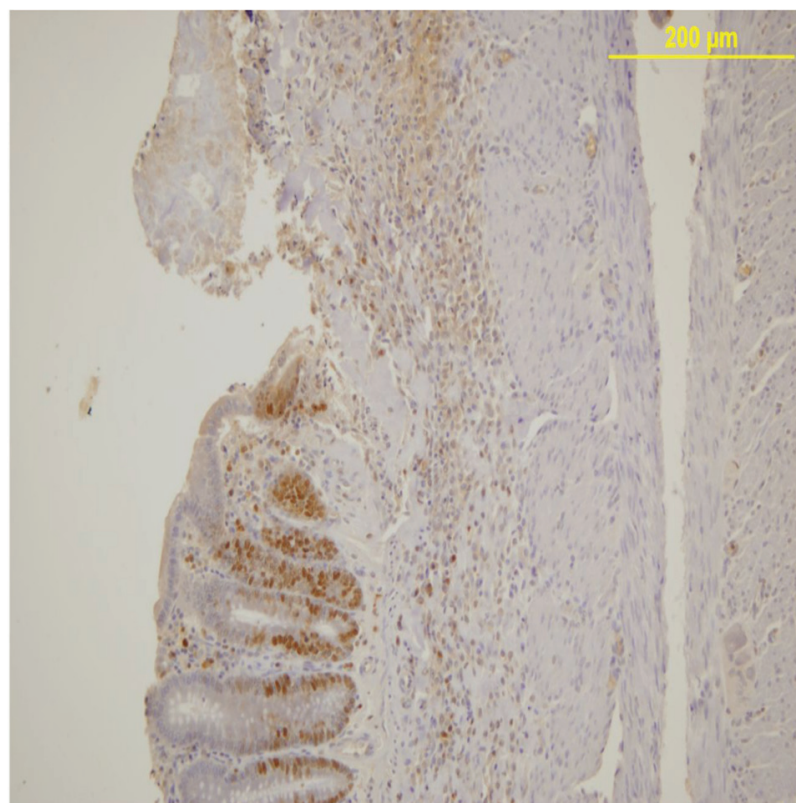

Figure 3. PCNA expression in the mucosal crypts adjacent to the ulcer. Immunoperoxidase staining. Original magnification, $\times 100$. mean macroscopic and histological scores (Fig. 1).

As shown in Figure 2, PCNA levels were significantly higher in group 1 and group 2 compared to the control group (66.6 \pm 23.4 , $59.0 \pm 21.1$ vs. $30.4 \pm 11.8, \mathrm{P}=0.018, \mathrm{P}=0.047$, respectively)

There were no statistically differences found between group 1 and group 2.

PCNA expression analysis, detected by immunoperoxidase, in the epithelial cells of the mucosal crypts is shown in Figures 3 and 4.

\section{Serum cytokines results}

Correlation between the effects of group 2 on inflammation and increases in circulating leves of TNF- $\alpha$ and IL-1 $\beta$, were also determined. The mean serum TNF- $\alpha$ level was $10.15 \pm$

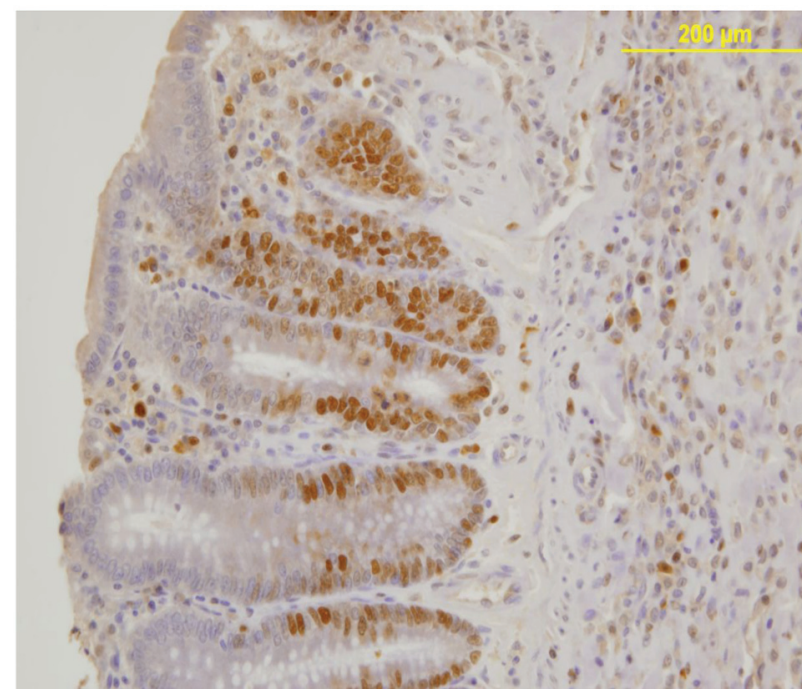

Figure 4. Nuclear PCNA expression in epithelial cells of the mucosal crypts. Immunoperoxidase staining. Original magnification, $\times 200$. 


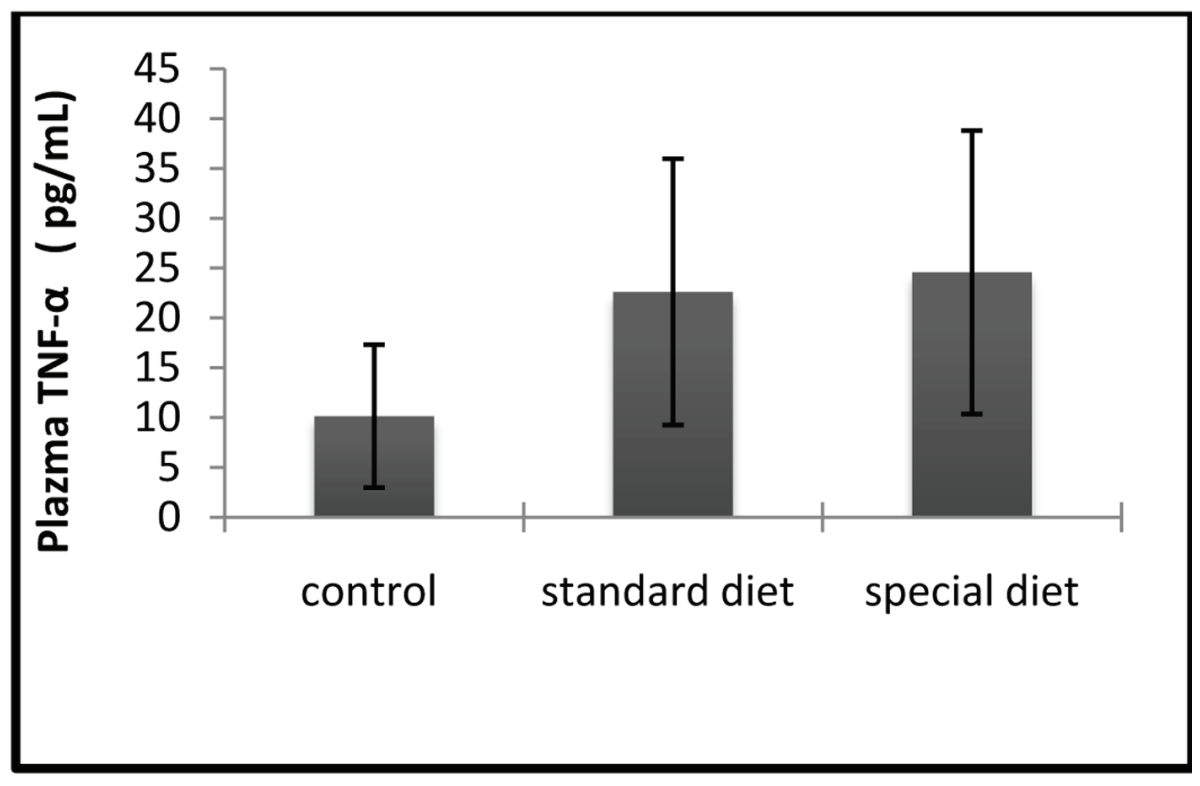

Figure 5. The serum TNF- $\alpha$ levels were comparable between groups. The results are shown as mean \pm SD.

$7.16 \mathrm{pg} / \mathrm{mL}$ in the control group, $22.61 \pm 13.36 \mathrm{pg} / \mathrm{mL}$ in group 1 , and $24.58 \pm 14.23 \mathrm{pg} / \mathrm{mL}$ in group 2 (Fig. 5).

The mean serum IL-1 $\beta$ level was $9.71 \pm 5.86 \mathrm{pg} / \mathrm{mL}$ in the control group, $25.61 \pm 7.36 \mathrm{pg} / \mathrm{mL}$ in group 1 , and $28.82 \pm 9.31$ $\mathrm{pg} / \mathrm{mL}$ in group 2 (Fig. 6).

Statistically significant differences were not found in the treatment groups.

\section{EDEF and TBARS}

The ETT and TBARS levels in standard and special diet groups were significantly increased $(\mathrm{P}<0.05$; Fig. 7,8$)$.

The mean plazma TBARS level was $165.65 \pm 47.76$ $\mathrm{nmol} / \mathrm{g} \mathrm{Hb}$ in the control group, $223.61 \pm 33.36 \mathrm{nmol} / \mathrm{g} \mathrm{Hb}$ in group 1 and $210.58 \pm 9.72 \mathrm{in} \mathrm{nmol} / \mathrm{g} \mathrm{Hb}$ in group 2. Although the intestinal inflammation caused significant increases in the TBARS levels compared to control group, they did not return to control levels when the animals were placed on the special diet (Fig. 7).

The mean ETT level was $1.64 \pm 0.04 \mathrm{~ms}$ in the control group, $2.48 \pm 0.05 \mathrm{~ms}$ in group 1 and $2.26 \pm 0.05 \mathrm{~ms}$ in group 2 .

Although intestinal inflammation caused a significant increase in ETT compared to control group, this did not return

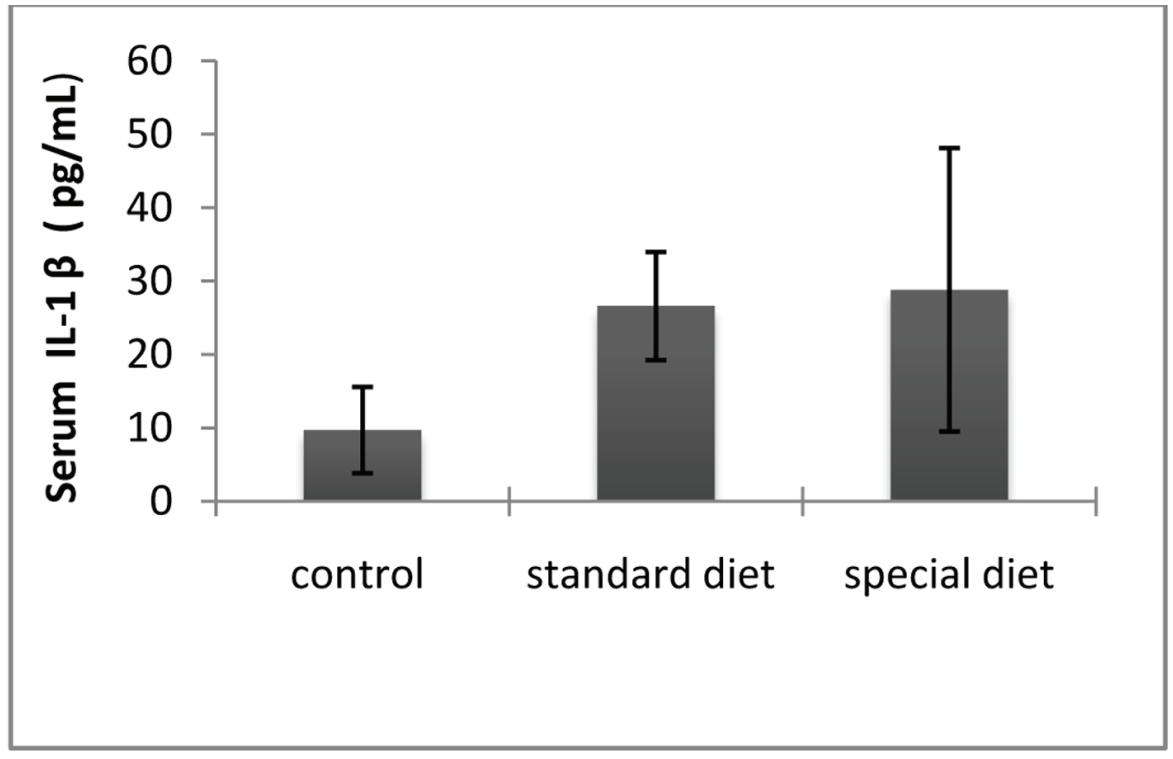

Figure 6. The serum IL-1 $\beta$ levels were comparable between groups. The results are shown as mean $\pm S D$. 


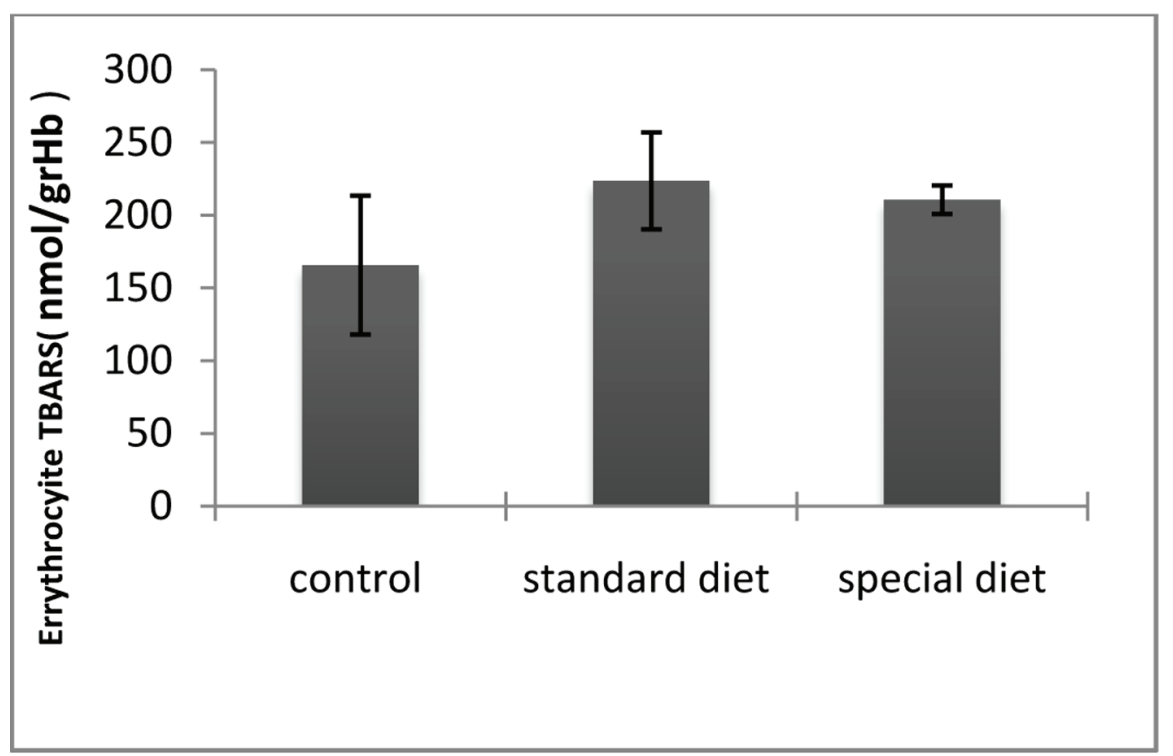

Figure 7. TBARS levels in erythrocytes. ${ }^{*} \mathrm{P}<0.05$ compared with the control group.

to the control level when the animals were applied a special diet (Fig. 8).

\section{Discussion}

There have been a fairly small number of studies directed toward the therapeutic use of Gln and omega-3 FA for the treatment of IBD. However very limited number of preclinical studies consistently supported a positive role of Gln as a therapeutic modality in IBD. In various models of chemicalsinduced colitis, treatments with enteral or local Gln and omega-3 FA were shown to improve a variety of clinical, cellular, pathohistological and biochemical endpoints of colitis.[4, 25].

Macroscopic and microscopic damage [25-27], enhanced epithelial apoptosis [28], impaired gut barrier function [29] reduced tissue antioxidant defense/enhanced oxidative stress [30-33] in addition to expression of proinflammatory mediators [25, 34].

There is only one clinical study showing the relationship between EDEF and pathophysiology of IBD in the literature [9]. However, data showing the effect of Gln and omega-3 FA on the relation between EDEF and IBD, could not be found.

In animals, it has been shown that Gln protects the intestinal mucosa in different models of IBD [29, 35]. The beneficial role of Gln in these experimental models of enterocolitis can be explained through different mechanisms [30], and it has po-

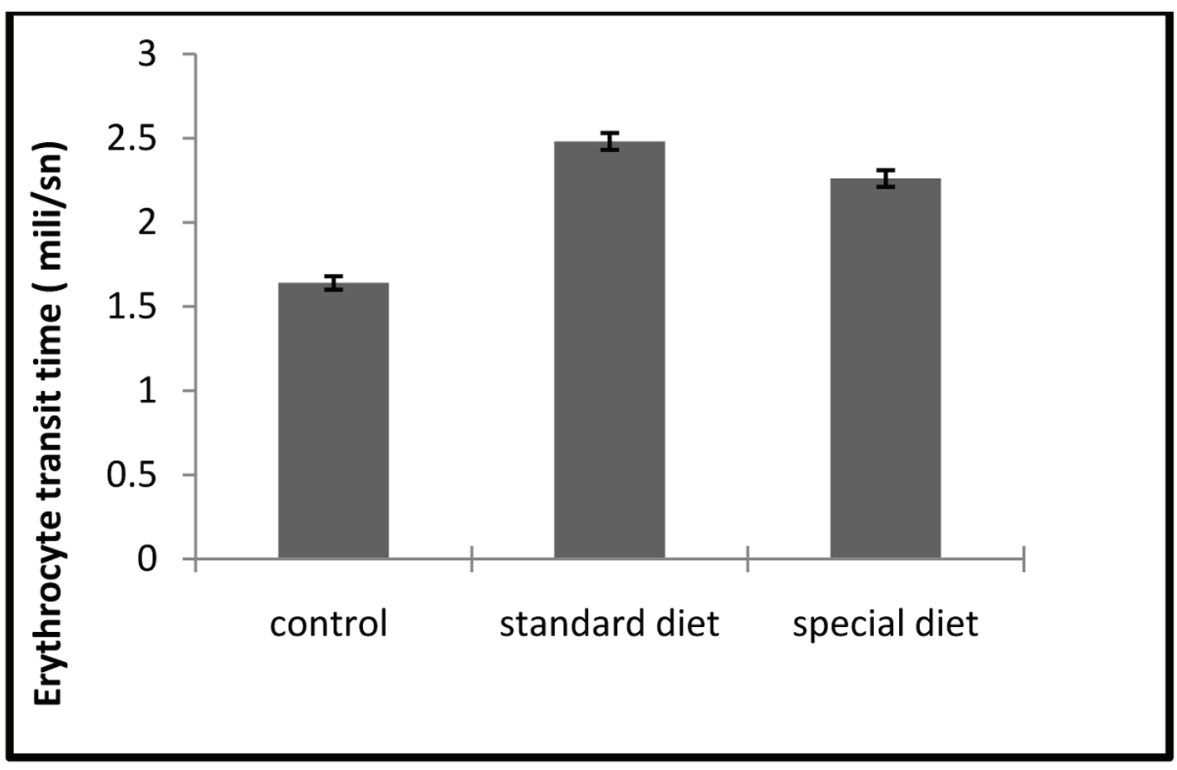

Figure 8. ETT assays. Each bar represents mean \pm SD. ${ }^{*} P<0.05$ compared to control group. 
tent antioxidant properties as well [36-38].

Antioxidant and anti-inflammatory effects induced by combination of omega-3 FA and Gln were expected to affect these mechanisms in our study, but the results of histopathological findings, and assessments of the levels of serum cytokines, erythrocyte TBARS and EDEF failed to reveal any beneficial effects of special diet.

Crohn's disease often affects the distal ileum and colon. Gln is the preferred fuel of enterocytes whereas butyrate is preferred by the colonocytes. Gln is absorbed in the proximal part of the small intestine and therefore, cannot reach to the inflamed part of the intestine [30]..But our knowledge in this regard is insufficient.

The route of administration and dose levels are also very important for the use of Gln since as excess amounts were found to have deleterious effects on ulcers in an experimental colitis model $[34,35]$. Because Shinozaki et al reported that excess Gln exacerbates toxic colitis in rats [38].

In our study, physiological dose and enteral route were used to induce a synergistic effects of Gln and omega-3 FA, but we did not observe any beneficial effects on macroscopic and histological scores. Also omega-3 FA promotes the production of less inflammatory eicosanoids from EPA and have other anti-inflammatory actions including decreases in leukocyte chemotaxis, adhesion molecule expression, $\mathrm{T}$ cell reactivity and inflammatory cytokine productions [39, 40].

The reduction of chemically induced colonic inflammation and damage by omega-3 FA has been demonstrated in numerous experimental animal models [19, 41-44]. Furthermore, there is strong indirect evidence supporting the potential of omega-3 FA in modulating intestinal inflammation [44].

Although we did not observe any beneficial effects on cytokine levels of (TNF- $\alpha$, IL-1 $\beta$ ), TBARS, macroscopic and histological scores with Gln and omega-3 FA in our studies. To determine the effectiveness of omega-3 FA in an animal model, we adopted an indomethacin-induced enterocolitis rat model in our present study. Ineffectiveness of omega-3 FA observed in our experiments may be due to use of a selected chemical to induce enterocolitis and the combined use of omega-3 FA with Gln which has been shown to have some deleterious effects in excess doses [38].

In our study, scoring of the small intestine was also performed, but in a similar study scoring of the colon should be included into the study, because IBD may involve both intestine and colon as seen in Crohn's disease.

Further aim of our study was to evaluate the in vivo IBD effects on EDEF and the oxidative damage marker TBARS.

Microvascular flow deceleration may play an important role in the etiopathogenesis of IBD. There are some findings that hypoperfusion and ischemia in the intestinal tissue of IBD patients are related to microvascular dysfunction [7]. The severity of intestinal injury has also been correlated with the degree of vascular injury [45-47]. Generated ROS are related to chronic inflammation that results in NF-kB activation. This also induces prolonged neutrophil infiltration and microvascular dysfunction and impairment of tissue oxygenation by increasing erythrocyte aggregation.

Elevated blood and plasma viscosity as well as impaired EDEF may produce retardation and stoppage of the flow of erythrocytes in microvessels.

Moreover, intensified erythrocyte aggregation resulting in subsequent local accumulation has been shown to be a key factor in microcirculation disruption [48]. There are also some studies reporting that ROS increase decrease in EDEF [8].

Only one clinical study of IBD has been reported to date. Akman et al showed that increased erythrocyte malonyldialdehyde values cause a reduction in EDEF [9]. It has been shown that increased TBARS levels can decrease EDEF in our study. In addition, we suggested that anti-inflammatory and antioxidant natures of Gln and omega-3 FA would exert protective effects on EDEF and TBARS levels, but we did not observe any statistically significant changes in these parameters in group 1 and group 2.

We believe that inadequate nutrient dose and duration of treatment may be not effective in our treatment groups. However, we were also unable to assess our results in the context of other reports, because there has been no previous study of EDEF in a experimental enterocolitis model.

Further studies will thus be required to clarify this issue. There is a need to specifically monitorize effects of nutrients on plasma erythrocytes.

In conclusion, we did not observe the expected synergistic effects antioxidant and anti-inflammatory effects of a combined use of Gln and omega-3 FA on EDEF in an indomethacin-induced enterocolitis rat model. Various causes may account for the observed disappointment results.

Initial, the doses of omega-3 FA and Gln used might be too low (or nonpharmacologic) to reveal a clinical therapeutic effect. Consistently, positive results obtained in the study of critical illness have used higher "pharmacological" Gln and omega-3 FA doses that exceed the usual physiological requirements, but it would be difficult to identify which is the optimal dose of omega-3 FA and Gln.

Finally, further studies are required for the determination of optimal route of administration and doses for Gln and omega-3FA and to clarify EDEF, erythrocyte aggregation and microvascular changes in inflammation.

\section{Grant}

The study was supported by an operating grant from the Dokuz Eylul University Research Foundation (grant number: 98/ KGB.SAG).

\section{Conflict of Interest}

The authors decleared no financial, commercial conflict of interest.

\section{References}

1. Schirbel A, Fiocchi C. Inflammatory bowel disease: Established and evolving considerations on its etiopathogenesis and therapy. J Dig Dis. 2010;11(5):266-276. 
2. Sands BE. Therapy of inflammatory bowel disease. Gastroenterology. 2000;118(2 Suppl 1):S68-82.

3. Yamamoto T, Nakahigashi M, Saniabadi AR. Review article: diet and inflammatory bowel disease--epidemiology and treatment. Aliment Pharmacol Ther. 2009;30(2):99112.

4. Calder PC. Fatty acids and immune function: relevance to inflammatory bowel diseases. Int Rev Immunol. 2009;28(6):506-534.

5. McClane SJ, Rombeau JL. Cytokines and inflammatory bowel disease: a review. JPEN J Parenter Enteral Nutr. 1999;23(5 Suppl):S20-24.

6. Hering NA, Schulzke JD. Therapeutic options to modulate barrier defects in inflammatory bowel disease. Dig Dis. 2009;27(4):450-454.

7. Hatoum OA, Heidemann J, Binion DG. The intestinal microvasculature as a therapeutic target in inflammatory bowel disease. Ann N Y Acad Sci. 2006;1072:78-97.

8. Piagnerelli M, Boudjeltia KZ, Vanhaeverbeek M, Vincent JL. Red blood cell rheology in sepsis. Intensive Care Med. 2003;29(7):1052-1061.

9. Akman T, Akarsu M, Akpinar H, Resmi H, Taylan E. Erythrocyte deformability and oxidative stress in inflammatory bowel disease. Dig Dis Sci. 2012;57(2):458-464.

10. Rajendran N, Kumar D. Role of diet in the management of inflammatory bowel disease. World J Gastroenterol. 2010;16(12):1442-1448.

11. Lucendo AJ, De Rezende LC. Importance of nutrition in inflammatory bowel disease. World J Gastroenterol. 2009;15(17):2081-2088.

12. Roth E. Nonnutritive effects of glutamine. J Nutr. 2008;138(10):2025S-2031S.

13. Fuchs BC, Bode BP. Stressing out over survival: glutamine as an apoptotic modulator. J Surg Res. 2006;131(1):26-40.

14. Wall R, Ross RP, Fitzgerald GF, Stanton C. Fatty acids from fish: the anti-inflammatory potential of long-chain omega-3 fatty acids. Nutr Rev. 2010;68(5):280-289.

15. Ruggiero C, Lattanzio F, Lauretani F, Gasperini B, Andres-Lacueva $\mathrm{C}$, Cherubini A. Omega-3 polyunsaturated fatty acids and immune-mediated diseases: inflammatory bowel disease and rheumatoid arthritis. Curr Pharm Des. 2009; 15(36):4135-4148.

16. Elson CO, Sartor RB, Tennyson GS, Riddell RH. Experimental models of inflammatory bowel disease. Gastroenterology. 1995;109(4):1344-1367.

17. Wallace JL, MacNaughton WK, Morris GP, Beck PL. Inhibition of leukotriene synthesis markedly accelerates healing in a rat model of inflammatory bowel disease. Gastroenterology. 1989;96(1):29-36.

18. Vilaseca J, Salas A, Guarner F, Rodriguez R, Martinez M, Malagelada JR. Dietary fish oil reduces progression of chronic inflammatory lesions in a rat model of granulomatous colitis. Gut. 1990;31(5):539-544.

19. Schwartz RS, Madsen JW, Rybicki AC, Nagel RL. Oxidation of spectrin and deformability defects in diabetic erythrocytes. Diabetes. 1991;40(6):701-708.

20. Solerte SB, Adamo S, Viola C, Carnevale Schianca GP, Crippa A, Ferrari E. Acute-phase protein reactants pattern and alpha 2-macroglobulin in diabetes mellitus. Patho- physiological aspects in diabetic microangiopathy. Ric Clin Lab. 1984;14(3):575-579.

21. Solerte SB, Adamo S, Viola C, Zambianchi E, Ferrari E. [Pentoxifylline and arterial hypertension in diabetes mellitus: long-term results in randomized groups]. Ric Clin Lab. 1985;15(Suppl 1):515-526.

22. Jain SK. Membrane lipid peroxidation in erythrocytes of the newborn. Clin Chim Acta. 1986;161(3):301-306.

23. Yamada T, Deitch E, Specian RD, Perry MA, Sartor RB, Grisham MB. Mechanisms of acute and chronic intestinal inflammation induced by indomethacin. Inflammation. 1993;17(6):641-662.

24. Stocks J, Dormandy TL. The autoxidation of human red cell lipids induced by hydrogen peroxide. Br J Haematol. 1971;20(1):95-111.

25. Ameho CK, Adjei AA, Harrison EK, Takeshita K, Morioka T, Arakaki Y, Ito E, et al. Prophylactic effect of dietary glutamine supplementation on interleukin 8 and tumour necrosis factor alpha production in trinitrobenzene sulphonic acid induced colitis. Gut. 1997;41(4):487-493.

26. Kretzmann NA, Fillmann H, Mauriz JL, Marroni CA, Marroni N, Gonzalez-Gallego J, Tunon MJ. Effects of glutamine on proinflammatory gene expression and activation of nuclear factor kappa B and signal transducers and activators of transcription in TNBS-induced colitis. Inflamm Bowel Dis. 2008;14(11):1504-1513.

27. Nieto N, Torres MI, Rios A, Gil A. Dietary polyunsaturated fatty acids improve histological and biochemical alterations in rats with experimental ulcerative colitis. J Nutr. 2002;132(1):11-19.

28. Vicario M, Amat C, Rivero M, Moreto M, Pelegri C. Dietary glutamine affects mucosal functions in rats with mild DSS-induced colitis. J Nutr. 2007;137(8):1931-1937.

29. Giris M, Erbil Y, Dogru-Abbasoglu S, Yanik BT, Alis H, Olgac V, Toker GA. The effect of heme oxygenase-1 induction by glutamine on TNBS-induced colitis. The effect of glutamine on TNBS colitis. Int J Colorectal Dis. 2007;22(6):591-599.

30. Israeli E, Berenshtein E, Wengrower D, Aptekar L, Kohen R, Zajicek G, Goldin E. Prophylactic administration of topical glutamine enhances the capability of the rat colon to resist inflammatory damage. Dig Dis Sci. 2004;49(10):1705-1712.

31. Fillmann H, Kretzmann NA, San-Miguel B, Llesuy S, Marroni N, Gonzalez-Gallego J, Tunon MJ. Glutamine inhibits over-expression of pro-inflammatory genes and down-regulates the nuclear factor kappaB pathway in an experimental model of colitis in the rat. Toxicology. 2007;236(3):217-226.

32. Scheppach W, Dusel G, Kuhn T, Loges C, Karch H, Bartram HP, Richter F, et al. Effect of L-glutamine and nbutyrate on the restitution of rat colonic mucosa after acid induced injury. Gut. 1996;38(6):878-885.

33. Arndt H, Kullmann F, Reuss F, Scholmerich J, Palitzsch KD. Glutamine attenuates leukocyte-endothelial cell adhesion in indomethacin-induced intestinal inflammation in the rat. JPEN J Parenter Enteral Nutr. 1999;23(1):1218.

34. Calder PC. Polyunsaturated fatty acids, inflammatory 
processes and inflammatory bowel diseases. Mol Nutr Food Res. 2008;52(8):885-897.

35. Xue H, Sufit AJ, Wischmeyer PE. Glutamine therapy improves outcome of in vitro and in vivo experimental colitis models. JPEN J Parenter Enteral Nutr. 2011;35(2):188197.

36. Coeffier M, Marion-Letellier R, Dechelotte P. Potential for amino acids supplementation during inflammatory bowel diseases. Inflamm Bowel Dis. 2010;16(3):518524.

37. Phanvijhitsiri K, Musch MW, Ropeleski MJ, Chang EB. Heat induction of heat shock protein 25 requires cellular glutamine in intestinal epithelial cells. Am J Physiol Cell Physiol. 2006;291(2):C290-299.

38. Shinozaki M, Saito H, Muto T. Excess glutamine exacerbates trinitrobenzenesulfonic acid-induced colitis in rats. Dis Colon Rectum. 1997;40(10 Suppl):S59-63.

39. Calder PC. Omega-3 polyunsaturated fatty acids and inflammatory processes: nutrition or pharmacology? Br J Clin Pharmacol. 2013;75(3):645-662.

40. Yates CM, Calder PC, Ed Rainger G. Pharmacology and therapeutics of omega-3 polyunsaturated fatty acids in chronic inflammatory disease. Pharmacol Ther. 2014;141(3):272-282.

41. Yuceyar H, Ozutemiz O, Huseyinov A, Saruc M, Alkanat M, Bor S, Coker I, et al. Is administration of n-3 fatty ac- ids by mucosal enema protective against trinitrobenzeneinduced colitis in rats? Prostaglandins Leukot Essent Fatty Acids. 1999;61(6):339-345.

42. Hudert CA, Weylandt KH, Lu Y, Wang J, Hong S, Dignass A, Serhan CN, et al. Transgenic mice rich in endogenous omega-3 fatty acids are protected from colitis. Proc Natl Acad Sci U S A. 2006;103(30):11276-11281.

43. Chapkin RS, Davidson LA, Ly L, Weeks BR, Lupton JR, McMurray DN. Immunomodulatory effects of (n-3) fatty acids: putative link to inflammation and colon cancer. $\mathrm{J}$ Nutr. 2007;137(1 Suppl):200S-204S.

44. Cabre E, Manosa M, Gassull MA. Omega-3 fatty acids and inflammatory bowel diseases - a systematic review. Br J Nutr. 2012;107(Suppl 2):S240-252.

45. Funayama Y, Sasaki I, Naito H, Fukushima K, Matsuno $\mathrm{S}$, Masuda T. Remodeling of vascular wall in Crohn's disease. Dig Dis Sci. 1999;44(11):2319-2323.

46. Ucar AD, Kocdor H, Canda AE, Unlu SM, Cehreli R, Kocdor MA. The effects of parenteral glutamine on intestinal adaptation in a rat model of short bowel syndrome. Cent Eur J Med. 2010;5(1):115-122.

47. Angerson WJ, Allison MC, Baxter JN, Russell RI. Neoterminal ileal blood flow after ileocolonic resection for Crohn's disease. Gut. 1993;34(11):1531-1534.

48. Lipowsky HH. Microvascular rheology and hemodynamics. Microcirculation. 2005;12(1):5-15. 